\title{
Substrate Mediated Long-Range Oscillatory Interaction between Adatoms: $\mathrm{Cu} / \mathrm{Cu}(111)$
}

\author{
Jascha Repp, ${ }^{1}$ Francesca Moresco, ${ }^{1}$ Gerhard Meyer, ${ }^{1,2}$ and Karl-Heinz Rieder ${ }^{1}$ \\ ${ }^{1}$ Institut für Experimentalphysik, Freie Universität Berlin, Arnimallee 14, D-14195 Berlin, Germany \\ ${ }^{2}$ Paul Drude Institut für Festkörperelektronik, Hausvogteiplatz, 5-7, D-10117 Berlin, Germany \\ Per Hyldgaard and Mats Persson \\ Department of Applied Physics, Chalmers University of Technology and Göteborgs University, S-41296, Göteborg, Sweden
}

(Received 2 June 2000)

\begin{abstract}
A quantitative study of the long-range interaction between single copper adatoms on $\mathrm{Cu}(111)$ mediated by the electrons in the two-dimensional surface-state band is presented. The interaction potential was determined by evaluating the distance distribution of two adatoms from a series of scanning tunneling microscopy images taken at temperatures of $9-21 \mathrm{~K}$. The long-range interaction is oscillatory with a period of half the Fermi wavelength and decays for larger distances $d$ as $1 / d^{2}$. Five potential minima were identified for separations of up to $70 \AA$. The interaction significantly changes the growth of $\mathrm{Cu} / \mathrm{Cu}(111)$ at low temperatures.
\end{abstract}

PACS numbers: 68.35.Fx, 61.16.Ch

Surface-state electrons on the close packed surfaces of noble metals form a two-dimensional nearly free electron gas. The scattering of the electrons off point defects and step edges generates standing wave patterns in the electron density, which can be directly observed with the scanning tunneling microscope (STM) [1]. Analysis of the standing wave patterns provides a direct way to determine the surface-state dispersion and the scattering properties of the scatterers $[2,3]$. The ability of single adatoms to scatter surface electrons can be used to confine electrons in so-called quantum corrals: artificial structures of single adatoms build up by atomic manipulation [4]. The previous studies on standing waves concentrated on the effects caused by the adatom scatterers on the surface electron gas. The twodimensional electron gas itself should, on the other hand, give rise to an interaction between the scatterers.

The surface-state mediated interaction is long ranged and oscillatory in nature. The history of indirect interactions mediated by the substrate electrons began with the theoretical works of Grimley [5], and Einstein and Schrieffer [6,7], followed by the experimental works of Tsong [8], and Watanabe and Ehrlich [9], who used field ion microscopy to observe the long-range interaction between single metal adatoms adsorbed on a W(110) surface. The long-range interaction mediated by a two-dimensional electron gas was considered in 1978 by Lau and Kohn [10]. They showed that, in the special case of a partially filled surface-state band, the interaction energy decays very slowly, as $1 / d^{2}$ for large separations and is oscillatory with a periodicity of half of the Fermi wavelength. Only recently a room temperature STM study discussed an indication of such a long-range interaction between strongly bonded sulfur atoms on a $\mathrm{Cu}(111)$ surface [11] and a few further qualitative investigations exist [12].

Here we report the first detailed quantitative study of a long-range interaction mediated by a two-dimensional nearly free electron gas. We have determined the inter- action energy between single $\mathrm{Cu}$ adatoms on a $\mathrm{Cu}(111)$ surface from extensive measurements of their mutual spatial correlations. Although the interaction is very weak, the very low diffusion barrier $(40 \mathrm{meV})$ [13-15] for the adatoms enabled us to probe their interaction energy up to distances of $70 \AA$. The distance dependence of the interaction shows an oscillatory behavior with a periodicity of half of the Fermi wavelength $\lambda_{F}$, and its envelope decays $\sim 1 / d^{2}$ for large separations $d$. We observe preferred adatom separations. The closest separation is $12.5 \AA$ and the others are larger by additional multiples of $\lambda_{F} / 2$. The main features of the observed interaction can be understood within an analytic theoretical model involving only the scattering properties of the adatoms and the band structure of the surface state [16]. A detailed understanding of this long-range interaction is very important, since its strength is large enough to dominate the growth of copper on $\mathrm{Cu}(111)$ at low temperatures. Apart from point defects, the long-range contribution also controls the interaction with step edges. This gives rise to a potential well and barrier formation in front of the step edges, which in turn results in an Ehrlich-Schwoebel-like barrier for attachment on both sides of the step edges.

Our experiments were performed with a low temperature STM [17] operated at 9-21 K. These temperatures are low enough to record the diffusion of single $\mathrm{Cu}$ adatoms on the close packed $\mathrm{Cu}(111)$ [15]. The sample has been cleaned by several sputter and anneal cycles. Bias voltages refer to the sample voltage with respect to the tip. As STM tips, we used electrochemically etched tungsten wires.

In Fig. 1(a), we show a STM image of the $\mathrm{Cu}(111)$ surface after evaporation of about 0.01 monolayer (ML) copper at $15 \mathrm{~K}$, a temperature where single copper adatoms are mobile. The image was taken later at $9 \mathrm{~K}$. Single copper adatoms, which appear as $0.4-\AA$-high protrusions and a few copper dimers with a height of $0.6 \AA$ can be observed. The copper adatoms form islands with a local hexagonal 

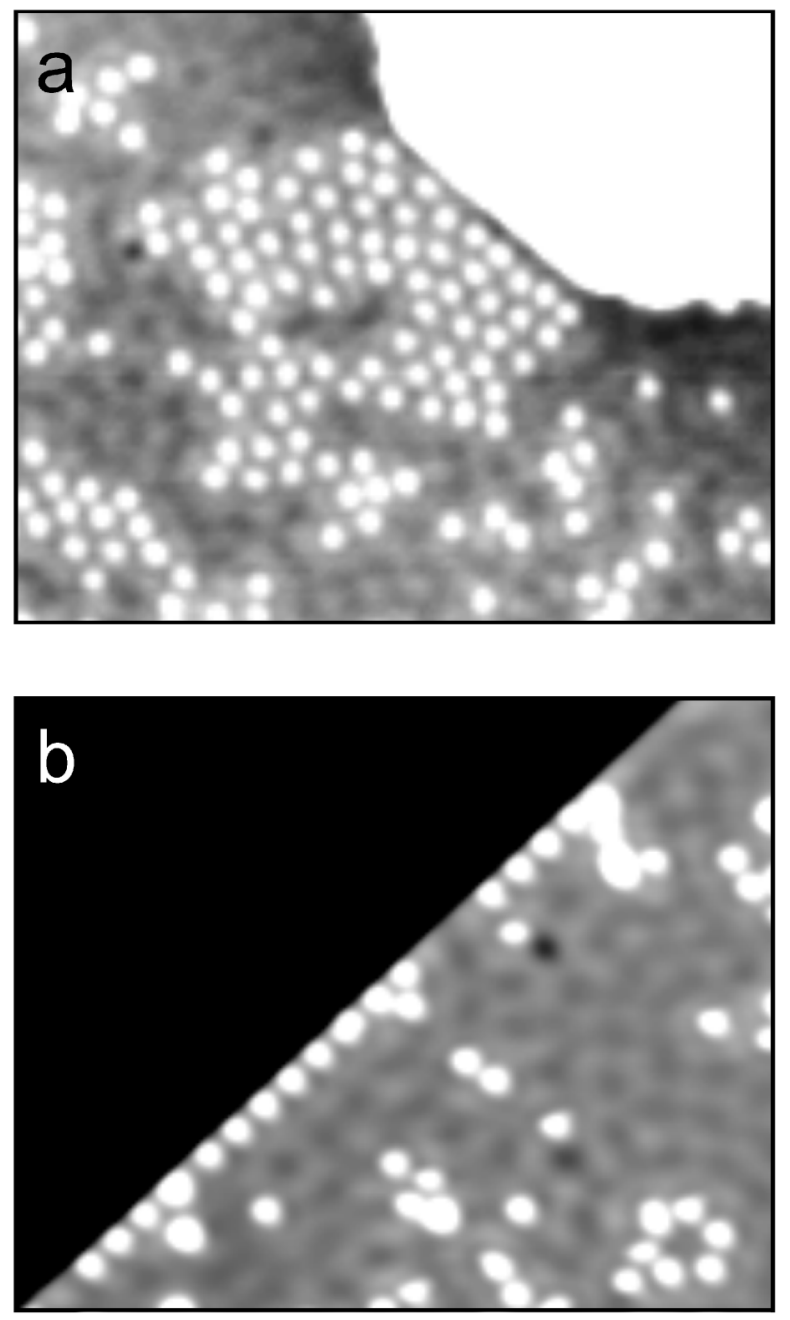

FIG. 1. Single $\mathrm{Cu}$ adatoms evaporated at $15 \mathrm{~K}$ on $\mathrm{Cu}(111)$. The STM images have been taken at $9 \mathrm{~K}$. (a) The $\mathrm{Cu}$ adatoms form an island with local hexagonal order and an average distance of $12.5 \AA$. The island is directly located at the lower side of a step edge, indicating a barrier for attachment to the step edge. Image size: $200 \AA \times 250 \AA$. Bias voltage: $100 \mathrm{mV}$. Current: $1.9 \mathrm{nA}$. (b) Single $\mathrm{Cu}$ adatoms are trapped in front of a descending step edge due to the long-range interaction. The average separation is $12.5 \AA$.

structure and a closest distance of about $12.5 \AA$. This indicates the existence of an attractive potential minimum at $12.5 \AA$ between the adatoms and a potential barrier for dimer formation. One island is located at the lower side of a copper step edge, as can be seen in the upper part of Fig. 1(a). This demonstrates the existence of a barrier for attaching single copper atoms to the lower part of the step edge. Also a barrier for the attachment to the upper part of the step edge can be clearly observed in Fig. 1(b). Such a barrier at the upper part of a step edge was also previously observed for $\mathrm{Pt} / \mathrm{Pt}(111)$ [18]. These images also show that the standing wave patterns due to the scattering of the surface-state electrons from the adatoms are on the same spatial scale as the observed correlations between the adatoms and suggest that these correlations might be generated by interactions mediated by these electrons.

To determine the interaction potential, $E(d)$, from the observed adatom-adatom correlations, we have used an analysis similar to the one applied previously by Tsong in field ion microscopy [8]. In thermal equilibrium at a temperature $T, E(d)$ is determined from the probability distribution, $g(d)$, to find an isolated adatom pair at a separation $d$. Here we determine $g(d)$ from the time dependence of the distance between two adatoms, sampled by taking STM images at constant time intervals. The variation in the binding energy of the considered sites is so small that they are occupied often enough to achieve excellent statistical information to extract $g(d)$. To measure possible tip effects we have determined, first, the hopping rate for different tunneling resistances, i.e., different tipadatom separations [19], and, second, we have varied the tip-adatom interaction time by taking image series with fixed scanning parameters but for different time intervals [20]. Figure 2(a) shows a histogram of the measured pair distribution, $g(d)$, of copper adatom distances extracted from a series of STM images. More than 65000 distances from a total of 3400 images were evaluated. The images were taken at a tunneling current of $200 \mathrm{pA}$ and a bias voltage of $300 \mathrm{mV}$. For these tunneling parameters the tip effects discussed above can be excluded. Furthermore one image was taken every $30 \mathrm{sec}$ at an adatom hopping rate of 0.1 hops/sec, ensuring sufficient statistical independence between subsequent images. The adatom coverage of $0.003 \mathrm{ML}$ was low enough to be in the low density regime [8]. One can clearly observe several equally spaced maxima and minima in the measured pair distribution.

To extract the interaction energy, $E(d)$, from the measured pair distribution, $g(d)$, we have to correct for two geometrical effects. First, the number of possible adatompair configurations increases linearly with separation $d$. This explains the strong increase for small distances [dotted curve in Fig. 2(a)]. Second, we have to consider the finite size of the measured STM images ( $115 \AA \times 115 \AA$ ), which results in a decrease of adatom pairs for large separations. In the case of a square-shaped image of width $a, g(d)$ has to be divided by the resulting geometric factor $P(d)=d\left[1-d(4 a-4 d+\pi d) / \pi a^{2}\right]$ [dashed curve in Fig. 2(a)]. $E(d)$ is finally calculated by $E(d)=-k T \ln [g(d) / P(d)]$ and is shown in Fig. 2(b).

The interaction energy, $E(d)$, is clearly oscillatory and up to five potential minima can be resolved, located at $12.5,27,41.5,56.5$ and $71 \AA$. The decay of the envelope of the interaction at larger separations is $\sim 1 / d^{2}$, as can be seen from a fit to the measured data represented by the dotted curve in the inset of Fig. 2(b). While only weak attractive minima (of maximum $-0.4 \mathrm{meV}$ ) are observed, the onset of a strong repulsion can be recognized for shorter distances than $10 \AA$. The above discussed equilibrium technique allows us to determine the potential energy [Fig. 2(b)] down to a distance of $7 \AA$. The 


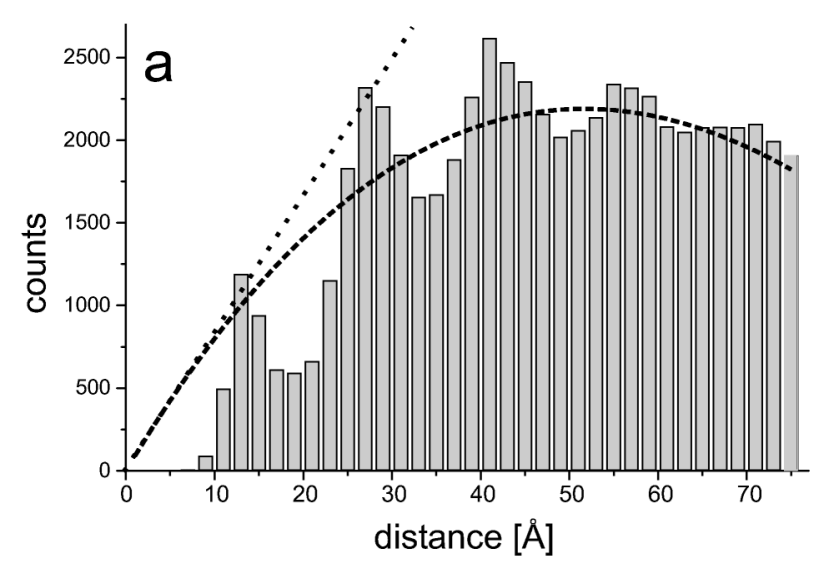

potential energy curve in Fig. 2(b), Figs. 3(a) and 3(c), respectively, the image shows a protrusion between the adatoms, whereas for the first maximum of this curve [Fig. 3(b)] the image shows a depression between the adatoms. A simulation of the STM images using a $s$ phase shift model for the scattering from the adatoms and Tersoff-Hamann theory shows that these protrusions and this depression comes from an increase and decrease of the LDOS, caused predominantly by single and double scattering events of the surface-state electrons from the adatoms.

In the theory by Hyldgaard and Persson [16] for the surface-state mediated interaction energy, the double scattering of the surface-state electrons from the adatoms at the Fermi level determines directly the one-electron energy term of the interaction energy at large distances. This theory provides a simple nonperturbative result for this interaction term in terms of the scattering phase shifts, given by

$$
E(d) \simeq-A\left(\delta_{F}, r\right)\left(\frac{4 \epsilon_{F}}{\pi^{2}}\right) \frac{\sin \left(2 q_{F} d+2 \delta_{F}\right)}{\left(q_{F} d\right)^{2}} .
$$

Here the dimensionless interaction strength $0<A<1$ is determined by $\delta_{F}$ and the reflection amplitude $r$ for the scattering of surface-state electrons is determined from a single adatom. The same functional form for the interaction energy was also obtained by Lau and Kohn [10] in their perturbative treatment but no direct relation to the scattering properties of the adatoms was provided. The three main criteria of the theory of surface-state mediated interaction energy are reproduced in the experimental data. The interaction energy is oscillatory with a period of $\lambda_{F} / 2$, the envelope of the magnitude decays as $1 / d^{2}$, and the phase shift is constant and does not change with separation $d$. We can further compare the experimentally determined interaction energy with the calculated one from Eq. (1) by using parameters corresponding to an ideal "black" scatterer, $\delta_{F}=\pi / 2$ and $r=0$, for which $A=0.25$. We find that the calculated interaction strength, $A=0.25$, is stronger than the experimentally determined strength, $A=0.08$, by a factor of 3 , and the calculated positions of the potential energy minima, 11, 25.5, 40, and $54.5 \AA$, are slightly smaller than the measured ones at 12.5 , $27,42.5$ and $57 \AA$. Although a phase shift of $0.3 \pi$ and $r=0.64$ would give an excellent fit to the measured data, these parameters are not easily reconciled with the measured scattering properties even when considering the experimental uncertainties in the determination of the phase shift and the potential energy curve.

A possible cause for the discrepancy between theory and experiments is the neglect of the electrostatic term to the interaction energy. This term can be shown to have the same functional form as the one-electron term but the calculation of its phase and strength is not as straightforward and also requires a description of the screening by the bulk electrons. 

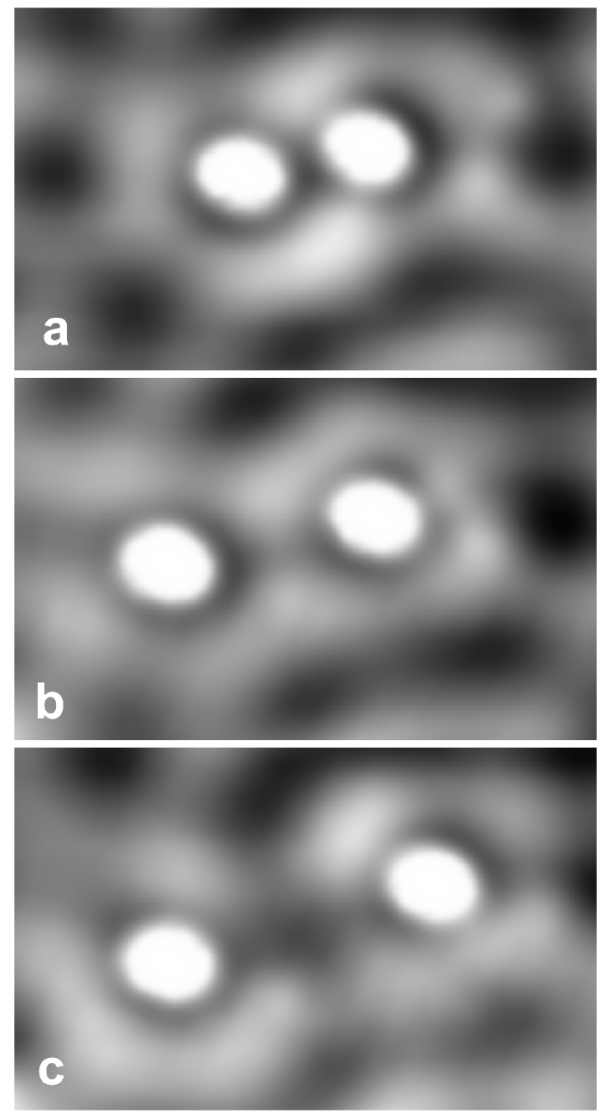

FIG. 3. STM images taken at three different adatom separations of (a) $12.9 \AA$, (b) $20.7 \AA$, and (c) $26.7 \AA$ corresponding, respectively, to a minimum, maximum, and minimum of the potential energy. The local density of states is decreased/increased in the case of a potential energy minimum/maximum between the adsorbates.

In summary, we have presented the first quantitative measurements on the long-range interaction between two single adatoms mediated by a two-dimensional electron gas. We observe clear oscillatory behavior of the potential energy with periodicity of $\lambda_{F} / 2$ and a separation dependence of $\sim 1 / d^{2}$. The interaction could be measured up to distances of $70 \AA$ corresponding to five potential minima. For adatom separations corresponding to potential minima/ maxima, we observe a decrease/increase in the local density of states at the Fermi level. The measured data were analyzed quantitatively by comparing them to a nonperturbative analytical estimate relating the potential energy behavior to the scattering properties of the adatoms.

We gratefully acknowledge K. Morgenstern and J.R. Manson for carefully reading the manuscript, and the European Union TMR project "Atomic/Molecular Manipulation" and the Deutsche Forschungsgemeinschaft Project No. RI 472/3-2 for partial funding. P. H. and M.P. are grateful for funding from the Swedish Natural Science Research Council (NFR) and the Swedish Foundation for Strategic Research (SSF) through the Materials Consortium No. 9.

[1] M. F. Crommie, C. P. Lutz, and D. M. Eigler, Nature (London) 363, 524 (1993).

[2] E. J. Heller, M. F. Crommie, C. P. Lutz, and D. M. Eigler, Nature (London) 369, 464 (1994).

[3] Ph. Avouris, Solid State Commun. 92, 11 (1994).

[4] M. F. Crommie, C. P. Lutz, and D. M. Eigler, Science 262, 218 (1993).

[5] T. B. Grimley, Proc. Phys. Soc. 90, 751 (1967).

[6] T.L. Einstein and J. R. Schrieffer, Phys. Rev. B 7, 3629 (1973).

[7] T. L. Einstein, in Handbook of Surface Science, edited by W. N. Unertl (Elsevier, New York, 1978), Vol. 1, p. 261.

[8] T. T. Tsong, Phys. Rev. Lett. 31, 1207 (1973); Rep. Prog. Phys. 51, 759 (1988).

[9] F. Watanabe and G. Ehrlich, Phys. Rev. Lett. 62, 1146 (1989).

[10] K. H. Lau and W. Kohn, Surf. Sci. 75, 69 (1978). A comment indicated how to generalize the perturbative approach of Lau and Kohn [T. L. Einstein, Surf. Sci. 75, L161 (1978)].

[11] E. Wahlström, I. Ekvall, H. Olin, and L. Wallden, Appl. Phys. A 66, S1107 (1998).

[12] M. N. Kamna, S. J. Stranick, and P. S. Weiss, Science 274, 119 (1996).

[13] W. Wulfhekel, N. N. Lipkin, J. Kliewer, G. Rosenfeld, L. C. Jorritsma, B. Poelsema, and G. Comsa, Surf. Sci. 348, 227 (1996).

[14] P. Stoltze, J. Phys. Condens. Matter 6, 9495 (1994).

[15] From temperature dependent diffusion measurements of isolated $\mathrm{Cu}$ adatoms we have determined a barrier height of $37 \pm 5 \mathrm{meV}$ and an attempt frequency of $5 \times 10^{13 \pm 1} \mathrm{~Hz}$.

[16] P. Hyldgaard and M. Persson, J. Phys. Condens. Matter 12, L13 (2000).

[17] G. Meyer, Rev. Sci. Instrum. 67, 2960 (1996).

[18] A. Gölzhäuser and G. Ehrlich, Phys. Rev. Lett. 77, 1334 (1996).

[19] T. R. Linderoth, S. Horch, E. Lægsgaard, I. Stensgaard, and F. Besenbacher, Phys. Rev. Lett. 78, 4978 (1997).

[20] K. Morgenstern, G. Rosenfeld, B. Poelsema, and G. Comsa, Surf. Sci. 352, 956 (1996).

[21] K. H. Lau and W. Kohn, Surf. Sci. 65, 607 (1977).

[22] L. Österlund, M. Ö. Pedersen, I. Stensgaard, E. Lægsgaard, and F. Besenbacher, Phys. Rev. Lett. 83, 4812 (1999).

[23] K. A. Fichthorn and M. Scheffler, Phys. Rev. Lett. 84, 5371 (2000).

[24] A. Bogicevic, S. Oresson, P. Hyldgaard, B. I. Lundqvist, H. Brune, and D. R. Jennison, Phys. Rev. Lett. (to be published). 